\title{
Energy Consumption Based Low Energy Aware Gateway (LEAG) Protocol in Wireless Sensor Networks
}

\section{A. Revathi, S. G. Santhi}

\begin{abstract}
Wireless Sensor Networks (WSN) consists of a large amount of nodes connected in a self-directed manner. The most important problems in WSN are Energy, Routing, Security, etc., price of the sensor nodes and renovation of these networks is reasonable. The sensor node tools included a radio transceiver with an antenna and an energy source, usually a battery. WSN compute the environmental conditions such as temperature, sound, pollution levels, etc., WSN built the network with the help of nodes. A sensor community consists of many detection stations known as sensor nodes, every of which is small, light-weight and portable. Nodes are linked separately. Each node is linked into the sensors. In recent years WSN has grow to be an essential function in real world. The data's are sent from end to end multiple nodes and gateways, the data's are connected to other networks such as wireless Ethernet. MGEAR is the existing mechanism. It works with the routing and energy consumption. The principal problem of this work is choosing cluster head, and the selection is based on base station, so the manner is consumes energy. In this paper, develop the novel based hybrid protocol Low Energy Aware Gateway (LEAG). We used Zigbee techniques to reduce energy consumption and routing. Gateway is used to minimize the energy consumption and data is send to the base station. Nodes are used to transmit the data into the cluster head, it transmit the data into gateway and gateway compress and aggregate the data then sent to the base station. Simulation result shows our proposed mechanism consumes less energy, increased throughput, packet delivery ration and secure routing when compared to existing mechanism (MGEAR).
\end{abstract}

Index Terms: WSN, LEAG, Nodes, Energy, MGEAR.

\section{INTRODUCTION}

WSN consists of a physical and environmental situation (temperature, pressure, a vehicle sensor, etc.,) and resource constraint problem (memory, energy and power consumption) there should be a resource constraints problem, such as of nodes in WSN. The sensors are powerful, monitoring and tracking the nodes. The WSN is processing among continuous monitoring and the remote system. WSN have concerned many researchers. It is used to connect wireless devices in the world. The WSN desires as many as advanced technologies to replace the design for wi-fi applications. WSN are self-design for a wireless ad-hoc network.

Revised Version Manuscript Received on Jun 20, 2019.

A.REVATHI, Pursuing Research, Computer Application, Annamalai University.

S. G. SANTHI, Assistant Professor in the Department of Computer Science and Engineering,

engineers and designers such as mechanical, electrical,
The major research areas are depending upon hardware and Operating System. WSN is a dynamic topology, application-oriented, network design, and nodes transmission[1]. hardware, and software engineers, etc.,

The Sensor solves many problems in network connections. In the wireless network, every and every node is linked into the Sensor Networks. The faraway thought is differing from a number of Wireless geographical location and measurement. WSN monitors power consumption, speed, and battery power. WSN introduce advanced technologies, such as Bluetooth, Wi-Fi, 2G, 3G, 4G, 5G,etc.,

WSN has been developed to execute the high level of transmitting information. There should be few sink nodes and many sensor nodes in the WSN. The storage capability and energy is small-scale capacity[2].

A WSN is used for wireless data connection to link the nodes. The WSN are computing system based networks that are used to link the nodes. It notifies the bandwidth utilization.

Sensor nodes are a powerful aggregation of distributed sensing, computing the devices. The sensor modifies a large number of application software and devices. WSN are especially interacting with the device and data transfer. Some devices can be connecting the signals from node to node transmission. It is a group of small random devices. WSN have the ability to display physical and environmental conditions.

\section{A. Zigbee}

ZigBee is an international standard wireless technology developed to use low energy digital local area signals for personal area networks. ZigBee operates on the IEEE 802.15.4 design and is used to create networks that require a short statistics transfer rate, energy efficiency, and tightly closed networking.

This technology is to increase productivity with low cost, securely processing and convenience network. System cost is low in the wireless medium. Thousands of sensors are connected into the small space. Zigbee is in popular concerned with the low cost and amplify energy efficiency. It implements the quantity of units such as MAC layer and Physical layer. It helps $250 \mathrm{kbps}$ and low latency.

This is low rate and objectives to furnish the Local Area Networks with low energy. It communicates all the shut by using devices. In Zigbee applications are used for geographical conditions, home automation, gaming, location tracking, etc. The Zigbee sensor networks consist of minimum energy to sense the devices and connecting the devices. The low latency of this technology is used to transmit the

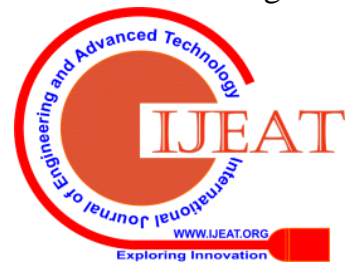


data. Zigbee is used for secure routing and connection. It is often used for Local Area Networks.

\section{EXISTING MECHANISMS}

\section{A. Leach}

Low Energy Adaptive Clustering Hierarchy (LEACH) is working to increase energy efficiency. It should be managing the nodes, selecting the cluster head randomly using a stochastic process which means randomly select the nodes as cluster head is the nearby base station at the same time the cluster head should connect the nodes into the round[3]. Each round comprises of a few nodes that are associated into the cluster head. Each round has the cluster head and gathering of hubs. The nodes send the information to the cluster head and cluster head total and compress the information send to the base station [4]. There are two phases.

$$
\begin{aligned}
& T(n)=\frac{P}{1-P \times\left(r \bmod P^{-1}\right)} \\
& T(n)=0 \\
& \forall n \in G \\
& \text { Where } \mathrm{n} \text { is a random number between } 0 \text { and } 1 \\
& P \text { is the cluster-head probability and } \\
& G \text { is the set of nodes that weren't cluster-heads the previous rounds }
\end{aligned}
$$

\section{B. M-Gear}

Multi-hop Gateway Energy Aware Routing protocol is sensor nodes have more sensed data for the base station to process[5]. Therefore, the regular technique of combine and aggregates the data into a simple set of significant data is required[6]. In order to develop the network lifetime and throughput, we organize a gateway node at the center of the network field. The gateway node is to gather the data from cluster head and from nodes near gateway aggregate and compress the data and sent to the base station. This process is to reduce the energy consumption gateway node[7].

\section{PROPOSED MECHANISM}

We proposed a novel based Protocol Low Energy Aware Gateway (LEAG). In this paper, we use the gateway to transmit the data into the base station. Every cluster head and the base station has the gateway to process the data(Figure.1). We insert the rechargeable gateway node because it is on position fact that the recharging of the gateway node is much better than the price of the sensor node. The round should be developed autonomously nodes and cluster head. Every round has the cluster head. The cluster head is selected autonomously based on location and distance of gateway and base station. The Zigbee is used to connect the nodes into the gateway. It also saves energy and reduces the cost of the networks. So the transmission and process should be good quality. LEAG reduce the energy consumption and secure routing. The gateway should process the cluster head and base station. Gateway is used to reduce the energy and routing. At the same time Zigbee techniques is a security routing. The LEAG performance is better than MGEAR.

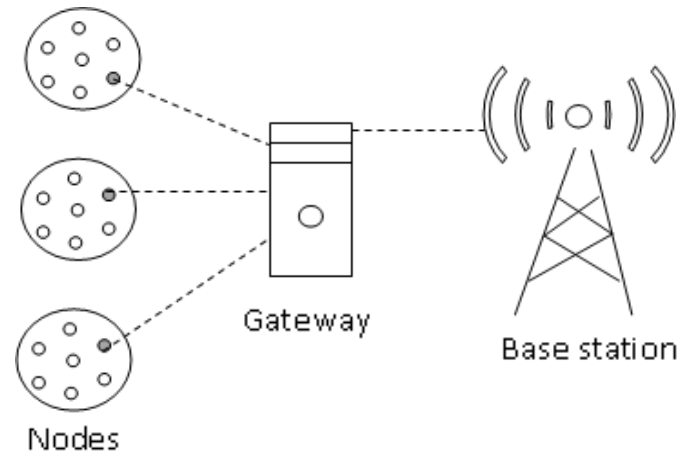

Figure. 1 The architecture of a LEAG protocol

A. Algorithm

1. Begin

2. Node creation

3. Cluster Head formation

4. Sending Advertisement to the gateway

5. Gateway stores the nodes distance and position $\mathrm{T}(\mathrm{S})=\left\{\frac{p}{1-P \times\left(\operatorname{rmod}\left(\frac{1}{P}\right)\right)} \quad \mathrm{s} \in C\right.$

6. Calculate the Distance $D=v T=E_{\text {given }} \frac{v}{P(v)}$.

7. Distance $\mathrm{x}$ is measured in units of $\mathrm{m}$, for meters.

8. Position: $\stackrel{\leftarrow}{\leftarrow}$ a vector in three dimensions described by a distance and a direction.

9. Throughput $=$ Number of packets received / Flow Time

10. End

B. Leag

In this paper, we applied the gateway techniques to transmit the data to the base station. Cluster head and the base station has the gateway to process the data (Figure.1). We insert the rechargeable gateway node because it is on position detail that recharging the gateway node. The round should be to develop autonomously nodes and cluster head. Each round has the cluster head. The cluster head is selected autonomously based on location and distance of gateway and base station. The Zigbee is used to connect the nodes into the gateway and base station. It also saves energy and reduces the cost of the networks. So the transmission and process should be good quality.

\section{Setup Phase}

In this phase, create the nodes and select the cluster head based on location and distance.

$$
\mathrm{T}(\mathrm{S})=\left\{\frac{p}{1-P \times\left(\operatorname{rmod}\left(\frac{1}{P}\right)\right)} \quad \mathrm{s} \in C\right.
$$

$\mathrm{P}=$ the desired percentage of $\mathrm{CHs}$ and $\mathrm{r}=$ the current round, $\mathrm{C}=$ set of nodes not elected as $\mathrm{CH}$ in the current round. The cluster head to base station direct connection has the maximum energy consumption when compare to gateway connection. The cluster heads are connected to the gateway. 


\section{Scheduling}

When all the sensor nodes are structured into clusters, each cluster heads creates TDMA based time slots for its member nodes. A 11 the associated nodes transmit their sensed data to cluster head in its own scheduled time slot. Something else, hubs change to an inactive hub. Hubs turn on their transmitters at the season of correspondence. Consequently, the vitality dispersal of the individual sensor hub diminishes.

\section{E. Steady-State Phase}

In the steady state phase, all sensor hubs transmit their detected information to the cluster head. The cluster head collects data from member nodes, aggregates and forwards to the gateway node. Gateway node receives data from cluster heads, aggregates and forwards to BS.

\section{F. Gateway Phase}

Gateway process reduces energy consumption. It stores the area and separation of the hub in the table that ought to be sent to the base station.

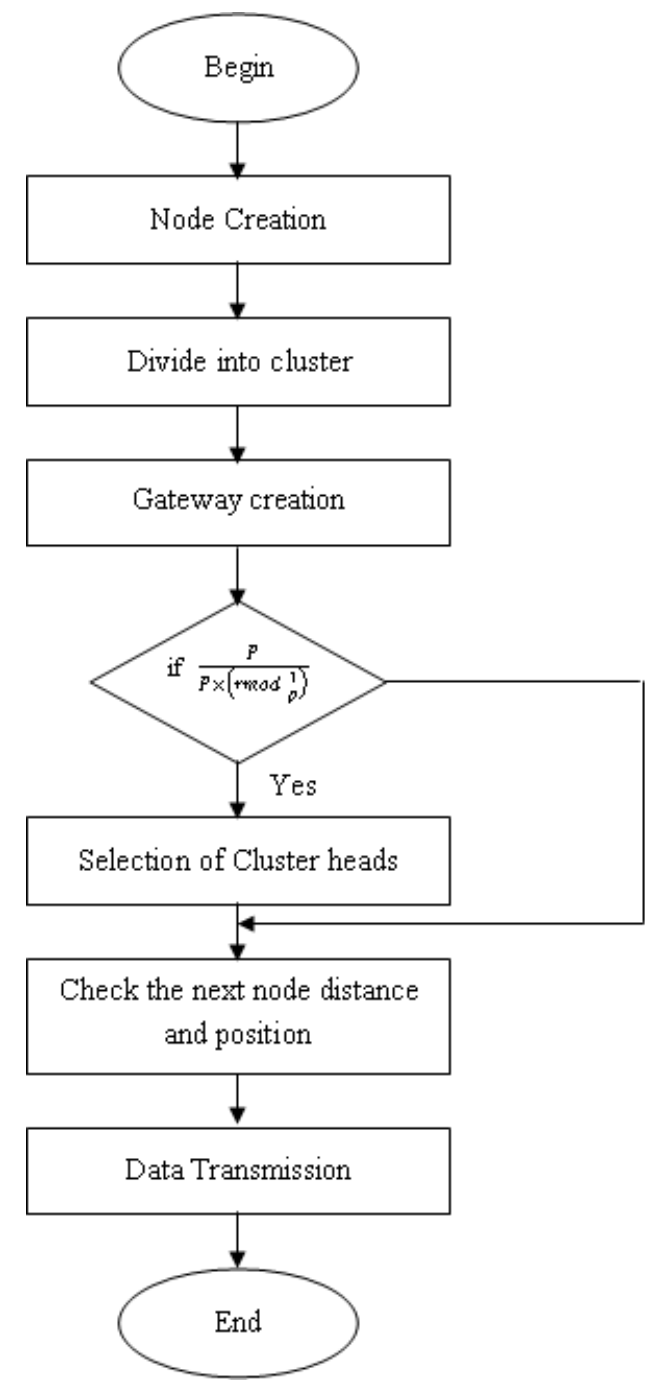

Figure.2. Flowchart for LEAG

\section{SIMULATION RESULTS}

The performance of a LEAG is used to reduce energy consumption. The nodes are sent through cluster head. The LEAG is analyzing the data result in packet transmission. The region inclusion and connection the hub between information is sent to the base station.

\section{A. Simulation Parameters}

\begin{tabular}{|l|l|}
\hline Parameter & Value \\
\hline Routing Protocol & AODV \\
\hline Area & $1000 * 1000$ \\
\hline Simulation time & $500 \mathrm{sec}$ \\
\hline Packet Size & 512 bytes \\
\hline Data traffic & CBR \\
\hline Bandwidth & $2 \mathrm{MBPS}$ \\
\hline Maximum speed & 5,10 and $15 \mathrm{~m} / \mathrm{sec}$ \\
\hline Number of Connections & $4,8,12$ and 16 connections \\
\hline Pause Time & 200 and 400 sec \\
\hline Packet generation rate & $2,3,4,5$ and 6 packets/sec \\
\hline
\end{tabular}

\section{B. Performance Metrics}

The LEAG is compared to MGEAR. The execution of the proposed work is superior to the current convention. The portal and cluster head hubs are to be an automatic procedure. The advantages of the proposed system are Packet delivery, Energy and Throughput.

\section{C.Packet Delivery Ratio}

The data are processed at a specific time. LEAG reduces the delay when compare to MGEAR. The gateways are to be an autonomous process. So the delay should be reduced.

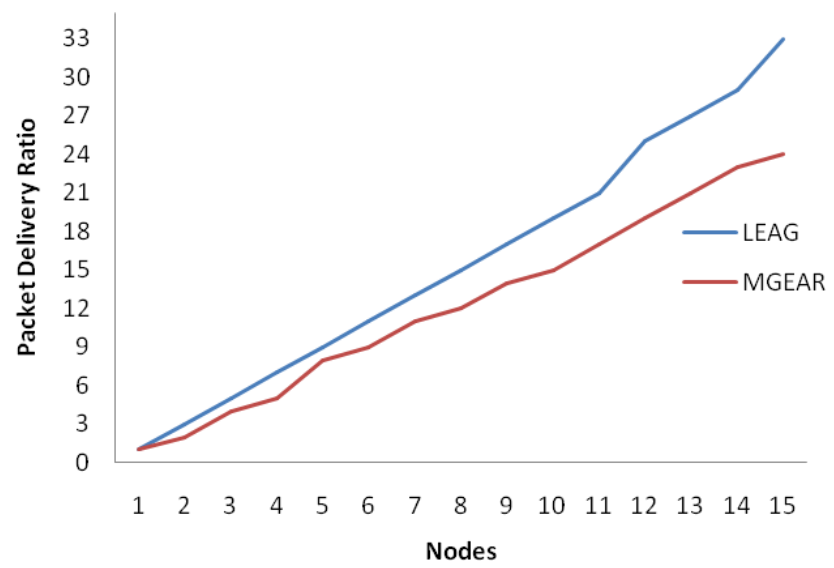

Figure.3. Packet Delivery Ratio

\section{D.Energy}

The Zigbee and proposed convention are utilized to standby 
energy. The nodes and cluster head sent the information to the gateway. The gateway process is to reduce the energy when contrasted with direct transmission. So they reduce energy utilization.

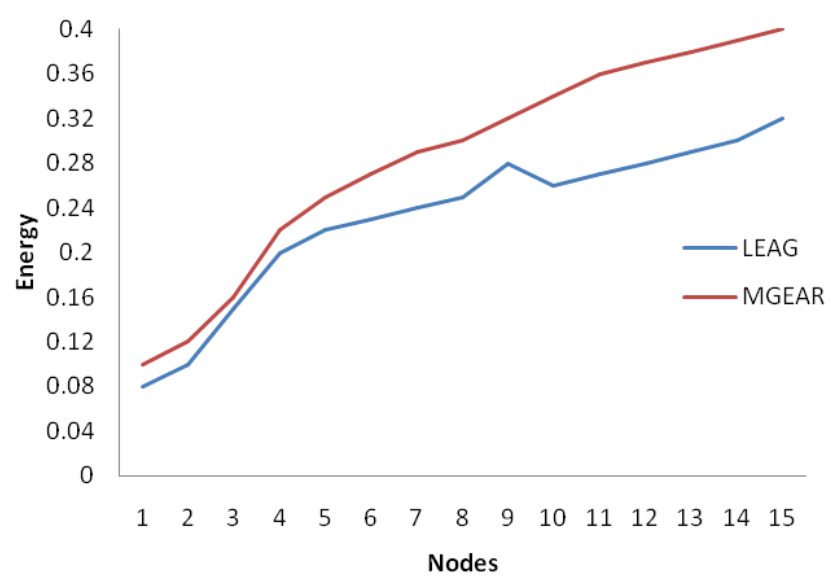

Figure.4. Energy Consumption

\section{E. Throughput}

The delay does not happen in the proposed LEAG protocol, at the same time the energy also saved. In this process, the throughput should be high when compare to MGEAR protocol.

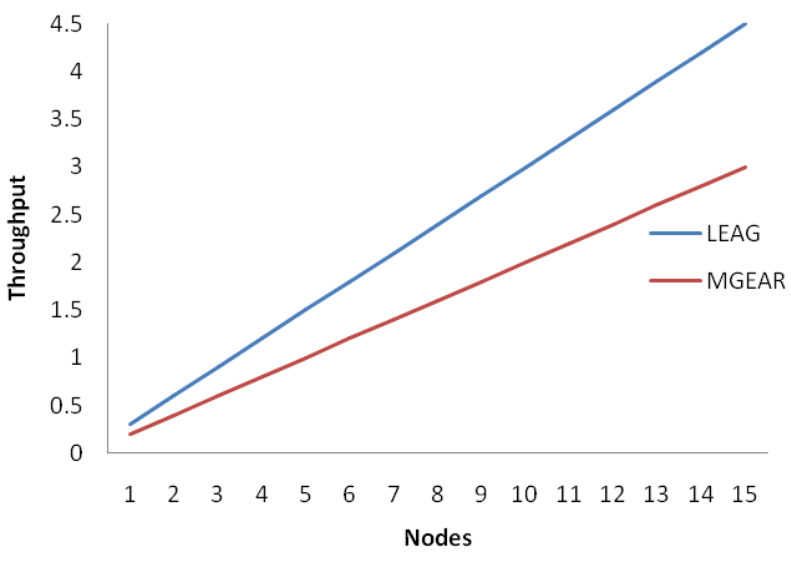

Figure.5. Throughput

\section{PROBLEM IMPLEMENTATION}

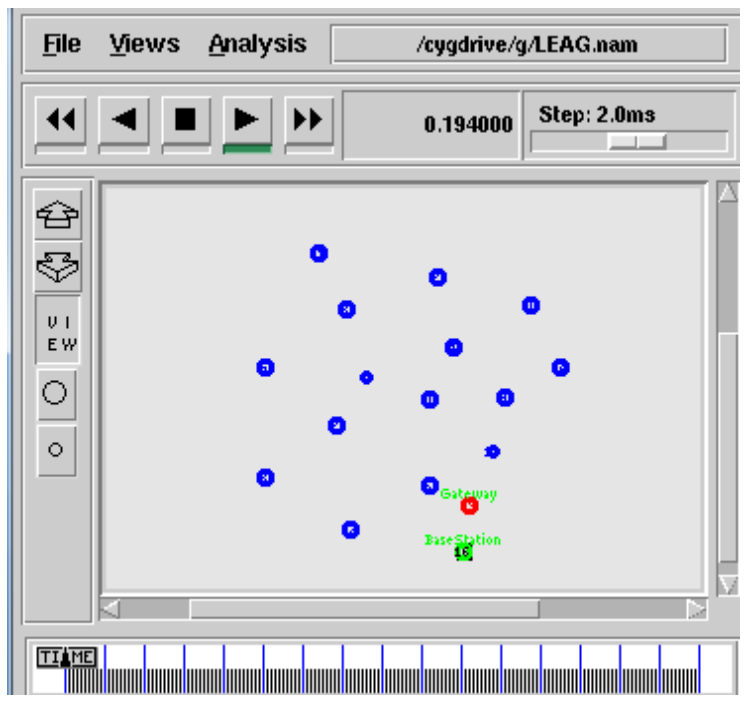

Figure.5. Nodes, Gateway, and Base station creation

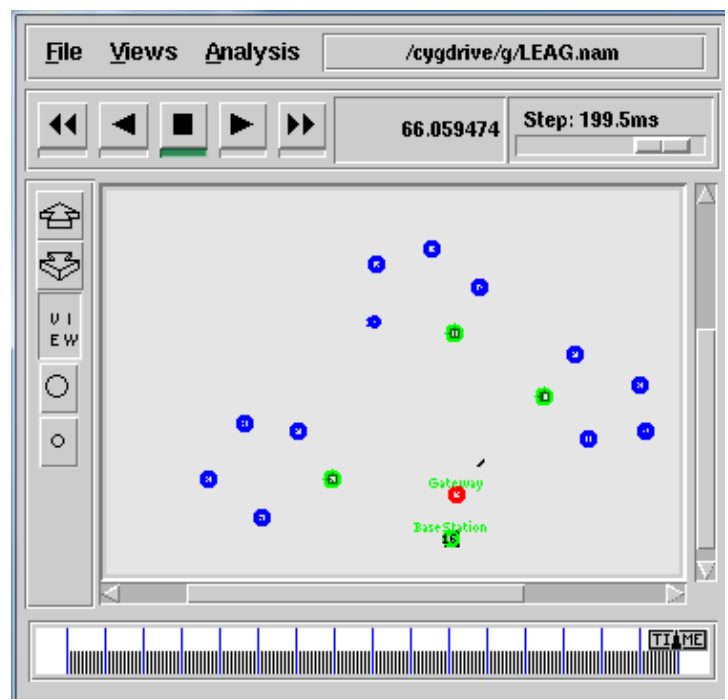

Figure.3. Data transmission

\section{CONCLUSION}

The sensors are based on energy, monitoring and tracking the nodes. The WSN is processing among continuous monitoring and the remote system. In WSN, LEACH is the basic energy saving clustering protocol and MGEAR is a routing and energy saving protocol. It is developed on LEACH. Monitoring vitality is a noteworthy standard for WSN. The gateway is the present principle. In this paper, we developed a hybrid novel based protocol LEAG. Energy consumption is reduced. The gateway technique is used to transfer the data via gateway nodes. The nodes are battery-powered. The gateway cost is low when contrasted with different nodes. The performance of the LEAG is reduced energy consumption and efficient routing.

\section{REFERENCES}

1. Dhaigude Tanaji Anand Rao, Dr. Latha Parthiban "A survey on Energy Efficient Routing Protocols" International Journal of Pure and Applied Mathematics, vol. 118, pp. 3109-3113, 2018.

2. Zhao Han, Jie Wu, Member, IEEE, Jie Zhang, Liefeng Liu, and Kaiyun Tian, A General Self-Organized Tree-Based Energy-Balance Routing Protocol for Wireless Sensor Network, IEEE Transactions on Nuclear Science,., vol. 61. April 2014, pp.732-740.

3. Amandeep Kaur, Er. Swaranjeet Singh, Navjot Kaur, "Review of LEACH Protocol and Its Types," in International Journal of Emerging Engineering Research and Technology, vol. 3, May 2015, pp. 20-25.

4. Monika, Sneha Chauhan, Nishi Yadav, "LEACH-I Algorithm for WSN" in International Journal of Innovative Research in Computer and Communication Engineering, vol.4, Issue..3, March 2016, pp. 3459 3466.

5. Amrit Singh, "A Novel Routing Protocol (M-Gear) Using Gateway Based Energy-Efficient Scheme For Wireless Sensor Networks (Wsns)", International Journal of Advanced Research in Computer Science, vol.7, issue.6, November 2016, pp. 361-363.

6. Hassan Oudani, Salahddine Krit, Mustapha Kabrane, Kaoutar Bandaoud, Mohamed Elaskri, Khaoula Karimi, Hicham Elbousty, Lahoucine Elmaimouni, "Energy Efficient in Wireless Sensor Networks Using Cluster-Based Approach Routing" International Journal of Sensors and Sensor Networks", International Journal of Sensors and Sensor Networks, vol.5, Issue.1, May 2017, pp.6-12.

7. Gurpreet Kaur, Sukhpreet Kaur, "Enhanced M-Gear Protocol for Lifetime Enhancement in Wireless Clustering System", International Journal of Computer Applications, vol.147, issue.14, August 2016, pp.30-34.

8. Kusum Lata, Kusum Dalal, "Performance Analysis of LEACH and M-GEAR Routing Protocols for WSN", International Journal of Electronics, Electrical and Computational System, vol.6, Issue.6, June 2017,pp. 164-169.

9. Neeraj Dewli, Mrs. Rashmi Saini, "A Comparative Analysis of M-GEAR and MODLEACH Energy Efficient WSN Protocols", International Journal of 
Computer Science and Information Technologies, vol.6, Issue.3, 2015,pp. 2641-2644.

10. Rahul Priyadarshi, Surender Kumar, SoniPrashant Sharma, "An Enhanced GEAR Protocol for Wireless Sensor Networks", Springer Nanoelectronics, Circuits and Communication Systems, vol.511,pp. 287-297.

11. Velanati Mohana Gandhi, M.V.H.Bhaskara Murthy, M.Lakshmu Naidu, "Performance Analysis of Multihop-Gateway Energy Aware Routing (M-Gear) Protocol for Wireless Sensor Networks", IOSR Journal Of Humanities And Social Science, vol.21,Issue.11,November 2016, pp.1-7.

12. Walid Abushiba, Princy Johnson, Saad Alharthi, Colin Wright, "An Energy Efficient and Adaptive Clustering for Wireless Sensor Network (CH-leach) using Leach Protocol" IEEE 13th International Computer Engineering Conference, December 2017, pp.50-54.

13. Mohammed Abo Zahhad, Sabah M. Ahmed, N. Sabor, and Shigenobu Sasaki, "Mobile Sink-Based Adaptive Immune Energy Efficient Clustering Protocol for Improving the Lifetime and Stability Period" IEEE Sensors Journal,Vol. 15, No. 8, pp 4576-4586, Aug 2015.

14. Gaurav Srivastav(2013), "Effective Sensory Communication using GEAR Protocol “ International Journal of Science and Research (IJSR) vol 9, issue 4, Pp 1809-1815.

15. Gaurav Srivastav(2013), "Effective Sensory Communication using GEAR Protocol “ International Journal of Science and Research (IJSR) vol 9, issue 4 , Pp 1809-1815.

16. C. Divya (2015), "Analysis of GFEAR Protocol "International Journal of Emerging Research in Management \&Technology, Volume-4, Issue-5, Pp 38-42.

\section{Authors Profile}

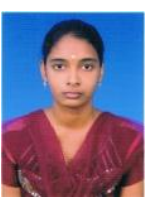

A.REVATHI obtained her Bachelor's degree in Computer Application from Shrimati Indira Gandhi College, Bharathidasan University, Tamilnadu, India in 2012.Then she obtained her Master's degree in Computer Application from Annamalai University, Tamilnadu, India in 2015. She is currently pursuing her Research Work in the Computer Application, Annamalai University. Her field of interest includes Wireless Sensor Networks.

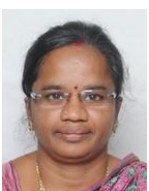

Dr.S.G.SANTHI obtained her Bachelor's in Computer Science and Engineering from Mookambigai College of Engineering, Bharathidasan University, Tamilnadu, India in 1992 and Master's in Computer Science and Engineering from Annamalai University, Tamilnadu, India in 2005. She pursued her Ph.D in Computer Science and Engineering at Annamalai University in 2015. She is currently working as an Assistant Professor in the Department of Computer Science and Engineering, Faculty of Engineering and Technology, Annamalai University. She has 2 decades of teaching experience in the Department of Computer Science and Engineering teaching both UG and PG courses. Currently she is guiding $4 \mathrm{Ph} . \mathrm{D}$ students. Her field of interest includes Wireless Sensor Networks, IoT, Web Security, Network Security. She has published more than 20 Research papers in International Journals, more than 10 papers in International Conferences and more than 10 papers in National Conferences. She is a life member in various professional bodies like ISTE, CSI, etc. 